International Journal of Pure and Applied Mathematics

Volume 108 No. 2 2016, 421-423

ISSN: 1311-8080 (printed version); ISSN: 1314-3395 (on-line version)

url: http://www.ijpam.eu

doi: 10.12732/ijpam.v108i2.16

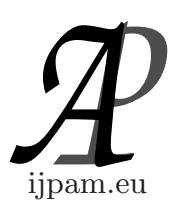

\title{
ON A PROBLEM OF MINIMAL NON-FC-GROUPS
}

\author{
Yıldız Aydın \\ Department of Mathematics \\ Faculty of Art and Science \\ Ondokuz Mayis University \\ Samsun, Turkey
}

\begin{abstract}
In this paper Problem 17.13 by A.O. Asar in The Kourovka Notebook is studied which is 'Let $G$ be a totally imprimitive $p$ - group of finitary permutations on an infinite set. Suppose that the support of any cycle in the cyclic decomposition of every element of $G$ is a block for $G$. Does $G$ necessarily contain a minimal non $-F C-$ subgroup?' and an example of a group $G$ satisfying these conditions but not having a minimal non $-F C-$ subgroup is given.
\end{abstract}

AMS Subject Classification: 20B35, 20F24, 20F05

Key Words: minimal non-FC-group, finitary symmetric group

\section{Introduction}

Let $\Omega$ be an infinite set and $\Omega_{1}, \Omega_{2}, \ldots$ be subsets of $\Omega$ such that $\bigcup_{i \in I} \Omega_{i}$, $\Omega_{i} \cap\left(\bigcup_{i \neq j} \Omega_{j}\right)=\emptyset$ and $\left|\Omega_{i}\right|=3$ for all $i$. Consider the group $G$ which is generated by $3-$ cycles of $\Omega_{i}$ 's. Clearly $G$ is a finitary permutation $3-$ group. Let us denote the $3-$ cycle of $\Omega_{i}$ by $\sigma_{i}$. Namely if $\Omega_{i}=\left\{\alpha_{i 1}, \alpha_{i 2}, \alpha_{i 3}\right\}$ then $\sigma_{i}=\left(\alpha_{i 1} \alpha_{i 2} \alpha_{i 3}\right)$ or $\sigma_{i}=\left(\alpha_{i 1} \alpha_{i 3} \alpha_{i 2}\right)$, it is not important which one you decide to be $\sigma_{i}$. For any pair of permutations $\sigma, \tau \in G, \sigma$ and $\tau$ are product of finitely many distinct $3-$ cycles. So $G \leq F \operatorname{Sym}(\Omega)$.

Received: February 16, 2016

Published: June 23, 2016
(C) 2016 Academic Publications, Ltd.

url: www.acadpubl.eu 


\section{Preliminaries}

Some definitions need to be given for this study. Firstly a support of a permutation $x$ is defined as $\operatorname{supp}(x)=\left\{\alpha \in \Omega \mid \alpha^{x} \neq \alpha\right\}$. A group $G$ is said to be finitary permutation group if every element of $G$ has finite support. A subset $\Delta$ of $\Omega$ is called a block for a group $G$ if $\Delta^{x}=\Delta$ or $\Delta^{x} \cap \Delta=\emptyset$ for all $x \in G$. Thus $\Omega$ and $\{\alpha\}$, for every $\alpha \in \Omega$, are blocks for $G$. These blocks are called proper blocks. If $G$ has no blocks except proper blocks $G$ is called primitive, otherwise $G$ is called imprimitive group. For an imprimitive group $G$, if there exists an infinite strictly ascending sequence of finite blocks, $G$ is said to be totally imprimitive group.[2] A group $G$ is called an $F C$-group if every element of $G$ has finitely many conjugates in $G$ and a group $G$ is called minimal non - FC-group if $G$ is not an $F C$ - group but which each proper subgroup is an $F C-$ group.[3].

\section{On Solution}

Theorem 1. Support of any cycle in the cyclic decomposition of every element of $G$ which is constructed above, is a block for $G$.

Proof. Let any pair of permutations $\sigma, \tau \in G$ are written in cyclic decomposition as follows: $\sigma=\sigma_{1} \ldots \sigma_{n}$ and $\tau=\tau_{1} \ldots \tau_{m}$. Of course $\sigma_{i}$ and $\tau_{j}$ are $3-$ cycles for every $1 \leq i \leq n$ and $1 \leq j \leq m$. To show that $\operatorname{supp}\left(\sigma_{i}\right)$ is a block for $G$ we need to analyze that in two possible cases: Case 1: $\operatorname{supp}\left(\sigma_{i}\right) \bigcap \operatorname{supp}\left(\tau_{j}\right)=\emptyset$ for all $1 \leq i \leq n$ and $1 \leq j \leq m$. In this case since any element of $\operatorname{supp}\left(\sigma_{i}\right)$ does not appear in any cycle of $\tau,\left(\operatorname{supp}\left(\sigma_{i}\right)\right)^{\tau}=\operatorname{supp}\left(\sigma_{i}\right)$ for every $1 \leq i \leq n$. Case 2 : At least one cycle contains same elements both in $\sigma$ and $\tau$ (at least one cycle in $\sigma$ and $\tau$ has same support). It is enough to prove it for one cycle. Let $\sigma_{i}$ and $\tau_{j}$ have same support in $\sigma$ and $\tau$. Hence every element of $\operatorname{supp}\left(\sigma_{i}\right)$ is moved by $\tau$, especially by $\tau_{j}$. But $\operatorname{supp}\left(\sigma_{i}\right)$ is fixed setwise by $\tau$. So $\left(\operatorname{supp}\left(\sigma_{i}\right)\right)^{\tau}=\operatorname{supp}\left(\sigma_{i}\right)$. Finally support of any cycle in the cyclic decomposition of every element of $G$ , is a block for $G$.

Also we now see that for all cases, support of any cycle in the cyclic decomposition of every element of $G$, is fixed setwise.

Theorem 2. Any union of finitely many $\Omega_{i}$ is block for $G$.

Proof. We concluded that every permutation in $G$ fixes the sets $\Omega_{i}$, for all $i$. Since $\Omega_{i} \cap \Omega_{j}=\emptyset$ for $i \neq j,\left(\Omega_{i} \cup \Omega_{j}\right)^{\sigma}=\Omega_{i}^{\sigma} \cup \Omega_{j}^{\sigma}=\Omega_{i} \cup \Omega_{j}$, for all $\sigma \in G$. 
So $\Omega_{i} \cup \Omega_{j}$ is a block for $G$.

Corollary 3. By adding different $\Omega_{i}$ to previous term we obtain an infinite strictly ascending sequence of finite blocks $\Omega_{1} \subset \Omega_{1} \cup \Omega_{2} \subset$... of $G$. So $G$ is a totally imprimitive group.

Proof. One can easily see that it is a conclusion of Proposition 3.2 .

Theorem 4. The group $G$, which is defined above is an $F C$-group.

Proof. Let $\sigma, \tau \in G, \sigma=\sigma_{1} \ldots \sigma_{n}$ and $\tau=\tau_{1} \ldots \tau_{m}$ in cyclic decomposition. We will prove it in similar way with proof of Proposition 3.1. Case 1: $\operatorname{supp}\left(\sigma_{i}\right) \cap$ $\operatorname{supp}\left(\tau_{j}\right)=\emptyset$ for all $1 \leq i \leq n$ and $1 \leq j \leq m$. In this case $\sigma^{\tau}=\sigma$ for all $\sigma, \tau \in$ $G$. Case 2: At least one cycle contains same elements both in $\sigma$ and $\tau$ (at least one cycle in $\sigma$ and $\tau$ has same support). We will prove it for one cycle. Let $\sigma_{i}$ and $\tau_{j}$ have same support in $\sigma$ and $\tau$. For simplicity we can write $\sigma=\sigma_{i} \sigma_{1} \ldots \sigma_{n}$ and $\tau=\tau_{1} \ldots \tau_{m} \tau_{j}$. Then we have $\sigma^{\tau}=\tau \sigma \tau^{-1}=\tau_{1} \ldots \tau_{m} \tau_{j} \sigma_{i} \sigma_{1} \ldots \sigma_{n} \tau_{j}^{-1} \tau_{m}^{-1} \ldots \tau_{1}^{-1}$. Since $\tau_{k}$ and $\sigma_{t}$ are distinct 3 -cycles for $k \neq j$ and $t \neq i$ we obtain $\sigma^{\tau}=$ $\tau_{j} \sigma_{i} \sigma_{1} \ldots \sigma_{n} \tau_{j}^{-1}=\tau_{j} \sigma_{i} \tau_{j}^{-1} \sigma_{1} \ldots \sigma_{n}$. Now $\sigma_{i}$ and $\tau_{j}$ are $3-$ cycles we have two subcases: Case 2.1: $\sigma_{i}=\tau_{j}$. In this case $\sigma^{\tau}=\tau_{j} \sigma_{i} \tau_{j}^{-1} \sigma_{1} \ldots \sigma_{n}=\sigma$, Case 2.2: $\sigma_{i}^{-1}=\tau_{j}$. In this case $\sigma^{\tau}=\tau_{j} \sigma_{i} \tau_{j}^{-1} \sigma_{1} \ldots \sigma_{n}=\sigma$. Anyone can easily see that $\sigma^{\tau}=\sigma$ for any pair of permutations $\sigma, \tau \in G$ which have one than more cycle contain same elements both in $\sigma$ and $\tau$ by induction. So $G$ is an $F C-$ group.

Corollary 5. $G$ does not have a minimal non-FC-Subgroup

Proof. Every subgroup of an $F C-$ group is an $F C-$ group by [1]. Hence $G$ does not have a minimal non $-F C-$ subgroup.

Someone can ask question what if $\left|\Omega_{i}\right|=p$, for some prime $p$. In this case $G$ would not be a $p-$ group. For example if $p=5$, though two 5 - cycles $\sigma=\left(\alpha_{1} \alpha_{2} \alpha_{3} \alpha_{4} \alpha_{5}\right)$ and $\tau=\left(\alpha_{1} \alpha_{2} \alpha_{4} \alpha_{3} \alpha_{5}\right)$ are belong to $G, \sigma \tau=\left(\alpha_{1} \alpha_{3}\right)\left(\alpha_{2} \alpha_{5}\right)$ is not a 5 -element so $G$ is not a $5-$ group.

\section{References}

[1] D.J.S. Robinson, A Course in the Theory of Groups, Springer-Verlag, New York (1982).

[2] J.D. Dixon, B. Mortimer, Permutation Groups, Springer-Verlag, New York (1996).

[3] M.J. Tomkinson, FC-Groups, Pitman Advanced Publishing Program, Boston-LondonMelbourne (1984). 
